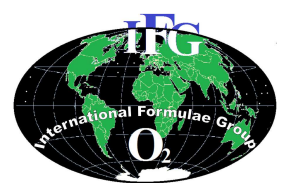

Available online at http://www.ifg-dg.org

Int. J. Biol. Chem. Sci. 10(3): 1173-1183, June 2016

International Joumal

of Biological and

Chemical Sciences

ISSN 1997-342X (Online), ISSN 1991-8631 (Print)

Original Paper http://ajol.info/index.php/ijbcs http://indexmedicus.afro.who.int

\title{
Analyse des systèmes de production de l'oignon bulbe autour du barrage de Goinré dans la province du Yatenga au Nord du Burkina Faso
}

\author{
Gouyahali SON*, Raymond KIOGO et Siédouba Georges YE \\ Institut de Recherche en Sciences Appliquées et Technologies, 03 BP 7047 Ouagadougou 03, Burkina Faso. \\ *Auteur correspondant ; E-mail : sgouyahali@yahoo.fr; Tél.: (226) 70245808
}

\section{RESUME}

L'oignon bulbe a intégré le marché sous-régional Ouest africain. Il représente 32,4\% des produits maraîchers au Burkina Faso. Afin d'améliorer sa compétitivité, une enquête prospective a été réalisée dans les principales zones de production du Burkina Faso. La présente étude vise à mieux connaitre les systèmes de production pratiqués et les contraintes techniques, à partir d'une grande région de production. Pour cela, des enquêtes et un suivi des opérations culturales ont été réalisés dans la zone de production ciblée. Les résultats ont révélé l'absence d'un cadre formel d'accès aux terres qui rend instable la production de ce légume. Toutes les opérations culturales étaient manuelles à l'exception du labour. La rareté de la main d'œuvre, à cause de la scolarisation et de la migration des jeunes vers les sites miniers, entraîne une augmentation des charges de production. Les opérations culturales les plus contraignantes sont l'émiettement/nivellement, le billonnage, le repiquage, le sarclage et le déterrage. L'utilisation des motopompes et le système d'irrigation à la raie engendrent des coûts de maintenance élevés, des risques de pannes fréquentes et des pertes importantes en eau dans les canalisations et à la parcelle. L'introduction d'équipements adaptés et le pompage solaire peuvent contribuer à l'amélioration du rendement et de la compétitivité de l'oignon du Burkina Faso.

(C) 2016 International Formulae Group. All rights reserved.

Mots clés: oignon, système de production, irrigation, opérations culturales contraignantes, coût de main d'œuvre, Burkina Faso.

\section{Analysis of onion bulb production systems near the Goinré's dam in the Yatenga province, Northern Burkina Faso}

\begin{abstract}
The onion bulb has been integrated into the West Africa sub-regional market. It accounts for $32.4 \%$ of vegetable production in Burkina Faso. In order to improve its competitiveness, a prospective survey was conducted on the main production zones. This study aims at better understanding the production systems and the technical constraints, based on one of the largest growing zone. For this purpose, surveys and a monitoring of the farming practices were carried out in the targeted production zone. The results revealed the absence of a formal framework for land access, which makes vegetable production unsecure. All the farming practices were manual except for ploughing. Labour scarcity due to schooling and migration of young people towards mining sites has increased vegetables production costs. The most constraining practices included crumbling/leveling, ridging, planting, weeding and unearthing. Motor pumps and furrow irrigation system generate high
\end{abstract}


maintenance costs, frequent breakdowns risks and significant water waste during irrigation and in the plot. The introduction of appropriate equipment and solar powered water pumping can contribute to improve onion production and competitiveness in Burkina Faso.

() 2016 International Formulae Group. All rights reserved.

Keywords: Onion, production system, irrigation, most constraining practices, labour cost, Burkina Faso.

\section{INTRODUCTION}

L'économie du Burkina Faso repose essentiellement sur l'agriculture comme l'atteste la contribution du secteur primaire à hauteur de 35 à $45 \%$ du PIB et qui absorbe près de $84 \%$ de la population active (Institut national des statistiques et de la démographie, 2003).

Le sous-secteur de la culture maraîchère est apparu dans les analyses, depuis les années 1990, comme une source de croissance agricole importante et de réduction de la pauvreté. En 2008, la production totale de spéculations maraîchères était évaluée à 747488 tonnes. La production maraîchère s'étend sur l'ensemble du territoire, dans toutes les provinces du pays. La production totale de l'oignon bulbe était de 242258 tonnes, soit $32,4 \%$ de la production totale maraîchère (Direction de la prospective et des statistiques agricoles et alimentaires, 2011). Une telle proportion confère à cette spéculation la première place parmi les produits maraîchers. Toutefois, l'oignon bulbe a intégré le marché international. En effet, si le Niger est le premier pays exportateur à l'intérieur de l'espace de la Communauté Economique des Etats de l'Afrique de l'Ouest (CEDEAO), suivi du Burkina Faso, ce marché est concurrencé par l'oignon bulbe en provenance des pays européens dont les PaysBas est le principal concurrent (Réseau national des chambres d'agriculture, 2011). Pourtant, la filière oignon est à l'origine de près de 400000 emplois, dont 100000 sont occupés par les femmes au Burkina Faso. Toutefois, les techniques de production restent toujours traditionnelles et les superficies exploitées varient entre 0,05 et 0,25 ha pour 90\% des producteurs (Direction générale des prévisions et des statistiques agricoles, 2008).
Les rendements sont variables d'une zone à l'autre, mais on note une nette évolution de 15 t/ha en 2005 à 21 t/ha en 2008, soit une progression d'environ $40 \%$ (Direction de la prospective et des statistiques agricoles et alimentaires, 2011). En dépit de cette évolution, il existe toujours une importante marge d'augmentation du rendement moyen, car les variétés cultivées au Burkina Faso ont un potentiel de rendement commercialisable d'environ 45 t/ha (Alessandro et Alseny, 2008). Il est donc primordial, d'améliorer les techniques culturales pour plus de compétitivité de ce produit sur le marché extérieur.

La présente étude vise à identifier les contraintes techniques prioritaires à lever pour contribuer à la compétitivité de l'oignon bulbe.

\section{MATERIEL ET METHODES}

Selon les enquêtes prospectives réalisées par Son et al. (2014) auprès des organisations des producteurs sur les principaux sites de production au niveau national, il ressort que la région du Nord est celle qui a les grandes superficies emblavées (Figure 1). Pendant la campagne 2007-2008, cette a fourni $61 \%$ de la production maraîchère nationale (Direction de la prospective et des statistiques agricoles et alimentaires, 2011). Aussi, selon la même source, la superficie totale exploitée dans cette région était de 2475,64 ha, soit $37 \%$ de la superficie de la production d'oignon bulbe au Burkina Faso. Cette production provenait essentiellement des provinces du Yatenga (37 355 tonnes) et du Zondoma (11 095 tonnes). La province du Yatenga fournit $77 \%$ de la production de la région du Nord avec $65 \%$ de superficies exploitées de cette région 
(Direction de la prospective et des statistiques agricoles et alimentaires, 2011).

Les échanges avec les responsables de la direction régionale de l'agriculture, la direction provinciale de l'agriculture, la Chambre Régionale d'Agriculture et certains projets comme le Projet d'Appui aux Filières Agro - Sylvo-Pastorales (PAFASP) ont permis de savoir que les plus grands sites de production sont aux abords du périmètre du barrage de Goinré dans la commune de Ouahigouya chef-lieu de la province du Yatenga.

Etant donné la similitude des pratiques dans les zones de production (Son et al., 2014), la présente étude a été réalisée autour du barrage de Goinré. La démarche méthodologique adoptée pour la réalisation de l'étude comporte les étapes suivantes:

- Identification de la plus grande région de production de l'oignon bulbe du pays;

- Identification des sites de production les plus performants et le choix des producteurs à enquêter;

- Enquête auprès des producteurs pour identifier les systèmes d'exploitation, les techniques et les coûts de production par opération;

- Suivi de l'itinéraire technique de production pour évaluer les temps de travaux au champ, les coûts de production ainsi que les outils utilisés et le genre.

Si l'étude bibliographique permet de choisir la plus grande région de production, l'identification des sites de production a fait l'objet d'une enquête réalisée auprès des responsables des services techniques de l'Etat, des projets et programmes, et des organisations faitières, intervenant dans la production maraîchère de la région. L'administration du questionnaire élaboré a permis de sélectionner les sites de production et les groupements de producteurs reconnus performants dans la production de l'oignon bulbe.

Une fois les groupements identifiés, un entretien semi structuré avec ces groupements a permis le choix des producteurs, sur la base des critères suivants:

- la précocité d'installation de la culture;

- le niveau de mécanisation du producteur;

- l'accessibilité de la parcelle;

- la disponibilité du producteur à accueillir les enquêteurs;

- le niveau de mécanisation et le genre.

Après le choix des producteurs à enquêter, un questionnaire semi-fermé leur a été administré, sur les systèmes et techniques de production, les coûts d'opération, ainsi que sur les équipements et les outils utilisés.

Le suivi des opérations de production a permis de vérifier d'une part, les informations obtenues lors de l'enquête auprès des producteurs et d'autre part, de mesurer les temps de travaux de chaque opération culturale, de dénombrer la main d'œuvre utilisée selon les opérations et le genre, d'identifier les outils de production utilisés et de collecter les coûts pratiqués par opération.

Pour le suivi des temps de travaux, il a été noté les temps de début et de fin d'opération, ainsi que les temps d'arrêt quel que soit le niveau de mécanisation. Le temps de travail a été calculé selon la formule suivante:

$\mathrm{T}_{0}=\mathrm{Tm}-\mathrm{Ta}$ en secondes, avec

$\mathrm{T}_{0}$ : temps effectif de travail,

$\mathrm{T}_{\mathrm{m}}$ : temps au champ,

$\mathrm{T}_{\mathrm{a}}$ : temps d'arrêt.

Les temps de travaux ont été mesurés à l'aide d'un chronomètre digital, dans chaque parcelle. Les dimensions des parcelles ont été mesurées à l'aide d'une corde de $100 \mathrm{~m}$ de long. La superficie So $\left(\mathrm{m}^{2}\right)$ a été calculée en fonction de la forme de la parcelle. Les temps de travaux ont été évalués en h/ha selon la formule suivante:

, avec:

- So est la superficie travaillée en mètres carrés et;

- To (mn) est le temps mis pour travailler la superficie So par $\mathrm{n}$ personnes.

Concernant les coûts d'opération, le suivi a permis de collecter des données sur les 
parcelles auprès des prestataires de service et des propriétaires de parcelles. Il s'agit du:

- coût de la main d'œuvre (par superficie ou par personne, selon les pratiques);

- nombre de personnes sur la parcelle et par opération;

- coût de la prestation mécanisée, s’il y a lieu.

Le coût de la main d'œuvre a été calculé par hectare selon la formule suivante:

Coût de la main d'œuvre par hectare et par opération $\mathrm{C}_{\mathrm{ha}}=10000 \times(\mathrm{Co} / \mathrm{So})$

Avec Co: coût de la superficie travaillée (So).

Cependant, les coûts de la main d'œuvre de certaines opérations (l'irrigation et fertilisation) n'ont pas fait l'objet de suivi. Ils ont été obtenus à travers les données de l'enquête. Il en est de même pour les coûts des intrants (produits phytosanitaires, engrais). L'irrigation et la fertilisation sont réalisées par la main d'œuvre familiale, et généralement par des enfants non rémunérés. Le manque de motivation engendre des pertes de temps énormes dans la réalisation de ces opérations.

Les données d'enquête ont été dépouillées, avant de réaliser les traitements statistiques à l'aide de l'application Excel version 2010. L'analyse des données qualitatives a été réalisée après un codage manuel. Les données quantitatives et les graphiques ont été traités également avec le même logiciel Excel 2010.

L'enquête a couvert 32 exploitations mais pour le suivi, la taille des échantillons a varié selon les opérations pour les principales raisons suivantes:

- L'installation tardive de la culture par rapport à la période de l'étude, en raison du retard des récoltes des cultures hivernales, car ce sont les mêmes parcelles qui servent en culture pluviale et en contre saison;

- La réalisation souvent simultanée des opérations par les producteurs suivis, sur un rayon d'action d'environ 0,5 à 1 kilomètre.
RESULTATS

Répartition des producteurs enquêtés en fonction du niveau d'instruction et de leur expérience

Il ressort de l'enquête qu'environ $31 \%$ des producteurs n'étaient pas alphabétisés sur 32 enquêtés (Figure 2). Toutefois, ces producteurs bénéficiaient de formations, dont l'alphabétisation, à travers l'appui des structures faitières pour leur permettre d'avoir accès à certaines informations techniques. Aussi, ils ont au moins 5 ans d'expérience dans la production de l'oignon.

\section{Répartition des producteurs en fonction de leur âge}

La production de l'oignon intéressait une population relativement jeune. De l'échantillon étudié, $84 \%$ des producteurs étaient dans une classe d'âge comprise entre 20 à 45 ans et $16 \%$ de plus de 45 ans. Les femmes représentaient $42 \%$ contre $58 \%$ d'hommes de notre échantillon. La production maraîchère constitue une activité de contre saison où les jeunes et les femmes sont les principaux acteurs.

\section{Typologie des producteurs en fonction du niveau de mécanisation}

Le niveau de mécanisation est défini sur la base de la source d'énergie utilisée pour réaliser une opération. En fonction de la source d'énergie utilisée, on distingue 4 types de producteurs: les producteurs équipés de la traction motorisée $(3 \%)$, ceux ayant la traction motorisée et la traction animale (18\%), les producteurs équipés uniquement en traction animale $(66 \%)$ et les producteurs en manuel (13\%). Toutefois, seul le labour est mécanisé aussi bien chez les producteurs équipés en traction animale que chez ceux équipés en traction motorisée.

\section{Organisation de la production}

Les organisations faitières et les groupements contribuent essentiellement à la formation de leurs membres et à l'approvisionnement en facteurs de 
production, selon les besoins exprimés. La commercialisation et l'irrigation ne sont pas ou sont peu contrôlées par ces organisations des producteurs.

\section{Statuts fonciers des parcelles de production}

L'acquisition des parcelles se fait selon 3 modes: l'accès de droit par lignée familiale (propriétaire), l'emprunt et la location. Les résultats de l'enquête indiquent que $69 \%$ des producteurs étaient propriétaires de terre, $22 \%$ des enquêtés exploitaient par emprunt et $6 \%$ faisaient la location. Il existait aussi des propriétaires de terre qui louaient des parcelles pour accroître leur superficie. Ils représentaient $3 \%$ des producteurs enquêtés.

Les producteurs équipés en traction motorisée avaient une superficie moyenne de $1170 \mathrm{~m}^{2} \pm$ 102. Ceux ayant la traction motorisée et équipés de traction animale avaient une superficie moyenne de 12 $433,33 \mathrm{~m}^{2} \pm 1$ 028. Par contre, les producteurs en traction animale avaient une superficie moyenne de $4486 \mathrm{~m}^{2} \pm 532$. Les exploitants en manuel avaient en moyenne $85 \mathrm{~m}^{2} \pm 5$.

\section{Principales techniques de production de l'oignon}

L'enquête a révélé que $91 \%$ des producteurs pratiquaient la technique en billons contre $9 \%$ pour la technique en planches. La technique en planches était pratiquée sur des superficies inférieures à 350 $\mathrm{m}^{2}$. La parcelle était subdivisée en planches de $1 \mathrm{~m}$ de large sur environ $10 \mathrm{~m}$ de long. Les dimensions sont généralement mesurées aux pas. Les travaux se font manuellement à l'aide de pioches appelées «sole» ou à la daba.

En technique de production sur billons, l'ordre chronologique des opérations était le suivant: le défrichage ou traitement herbicide, la pré-humidification, le labour, l'émiettement/nivellement, le billonnage, l'irrigation, le repiquage, l'épandage d'engrais, l'épandage du fumier, le sarclage, la récolte et le conditionnement du produit en sacs. Cette technique en billons consiste d'abord, à labourer toute la parcelle, avant de la subdiviser en bandes de dimensions de 50 $\mathrm{m}$ de long sur $7 \mathrm{~m}$ de large (appelée couche par les producteurs). La longueur de la couche est mesurée à l'aide d'une ficelle et la largeur est déterminée au moyen d'un tube PVC de 6 $\mathrm{m}$ plus un pas. C'est sur chaque couche que sont réalisés les billons et les raies d'irrigation. Les coûts des prestations de service sont négociés par couche dans la plupart des cas.

\section{Suivi des opérations}

Le suivi des opérations culturales a été fait auprès de 15 producteurs sur le labour, l'émiettement/nivellement, le billonnage, le repiquage et le sarclage et la récolte. Il a permis de mettre en évidence les opérations de production de l'oignon.

\section{Défrichage}

L'usage des herbicides vise à réduire les mauvaises herbes pour faciliter le labour ou avant le repiquage. L'herbicide spécifique de prélevée est le Pentimethaline EC. Cette opération est réalisée à la demande par un membre de la famille. Toutefois, le coût du traitement obtenu par l'enquête est de 23560 FCFA/ha \pm 1048 .

\section{Labour}

Le labour est la première opération de préparation du sol. Il est réalisé le lendemain de la pré-humidification, à l'état friable du sol. Les équipements utilisés sont: la charrue à soc en traction animale, le pulvériseur en traction motorisée, en lieu et place de la charrue à disques ou à socs. Les temps de travaux et le coût de la main d'œuvre ont varié en fonction de la source d'énergie. Ainsi, en traction motorisée (avec un tracteur de $60 \mathrm{cv}$ et $90 \mathrm{cv}$ ), le temps moyen de travail était de $3 \mathrm{~h} / \mathrm{ha} \pm 1$ sur 3 parcelles suivies, avec un coût moyen de $42857 \mathrm{FCFA} / \mathrm{ha} \pm 1355$. En traction bovine, le temps moyen de labour était de $14 \mathrm{~h} / \mathrm{ha} \pm 4$ pour 11 producteurs suivis, dont le coût moyen était de $28571 \mathrm{FCFA} / \mathrm{ha} \pm 8528$. 


\section{Emiettement/nivellement}

Aussitôt après le labour, l'émiettement/nivellement simultané est réalisé manuellement à l'aide de la daba. Au cours de cette opération double, les racines et autres débris solides indésirables sont éliminés. L'opération est réalisée aussi bien par les hommes que par les femmes. Le temps moyen de travail calculé était de $66 \mathrm{~h} / \mathrm{ha} \pm 9$, avec un coût moyen de 35714 FCFA/ha \pm 10 428. Toutefois, dans la pratique, le coût de la main d'œuvre était fixé par couche de superficie de $350 \mathrm{~m}^{2}$. Mais lorsque la superficie est inférieure à cette superficie standard, les prix sont négociés.

\section{Billonnage}

Le billonnage constitue la dernière étape de la préparation du sol. Il consiste à réaliser des billons sur lesquelles se fait le repiquage. La réalisation des billons permet d'obtenir en même temps les raies d'irrigation. Le billonnage est effectué uniquement par des hommes à l'aide de la daba. La largeur moyenne des billons était de $21,75 \mathrm{~cm} \pm 2,02$. L'écartement moyen mesuré entre lignes était de $16,9 \mathrm{~cm} \pm 1,27$ et celui entre les plants était de $10,99 \mathrm{~cm} \pm$ de 1,37 . Sur 7 producteurs suivis, le temps moyen était de $76 \mathrm{~h} / \mathrm{ha} \pm 10$ et le coût moyen de la main d'œuvre était de 39796 FCFA/ha \pm 9088.

\section{Irrigation}

Le barrage de Goinré est la principale source d'eau utilisée par les producteurs. Il existe aussi quelques puisards qui sont utilisés pour l'arrosage des pépinières. Le système d'irrigation à la raie est le plus pratiqué. La motopompe, principal moyen d'exhaure de l'eau, est gérée de façon communautaire par le groupement. Un membre du groupement joue le rôle d'opérateur. Ce dernier est chargé de faire fonctionner la motopompe et de distribuer l'eau sur les parcelles de chaque membre du groupement selon un planning convenu à l'avance. La fréquence d'irrigation était une fois tous les 3 jours durant toute la campagne. Cependant, en début de campagne, chaque producteur ayant fini le nettoyage des résidus de récolte sur sa parcelle, bénéficiait d'une pré-humidification avant les travaux de préparation du sol.

Les charges de fonctionnement de la motopompe étaient assurées par une caisse alimentée par les cotisations des membres. Cette caisse était gérée par le président du groupement ou la structure faitière. Le montant des cotisations était fixé par couche $\left(350 \mathrm{~m}^{2}\right)$ et était variable selon le groupement. Pour pallier les risques de pannes souvent fréquentes, il existait généralement une motopompe de secours. La charge moyenne du pompage était évaluée à 342857 FCFA/ha \pm 15450 pour une campagne. Cette variation élevée est liée au coût de fonctionnement des motopompes, selon les puissances utilisées. La main d'œuvre pour l'irrigation de la parcelle était assurée par les enfants ou les producteurs eux-mêmes. Cependant, le prix de référence fixé par couche de $350 \mathrm{~m}^{2}$ était de 21450 FCFA /ha \pm 1200 par campagne.

\section{Repiquage}

Le repiquage est une opération manuelle, réalisée essentiellement par les femmes dans l'après-midi pour profiter des basses températures. Les plants étaient repiqués sur les flancs de part et d'autre du billon, après une irrigation pour faciliter l'enfouissement des plants mais aussi pour réduire le stress hydrique. Sur 11 producteurs suivis, l'écartement moyen mesuré entre les lignes était de $16,9 \mathrm{~cm} \pm 1,27$ et entre les plants de $10,99 \mathrm{~cm} \pm 1,37$. Le temps moyen de repiquage était de $301 \mathrm{~h} / \mathrm{ha} \pm 6$. Le coût moyen de la main d'œuvre a été évalué à 51 038 FCFA/ha \pm 7393 .

\section{Fertilisation}

Des 5 formules de NPK utilisées que sont : 14-18-18 ; 15-15-15 ; 15-15-23 ; 14-2314 et 23-10-5 qui était la plus répandue, contre la formule d'urée perlée à $46 \%$. L'application manuelle de l'engrais chimique se faisait en deux étapes et par poquet auprès des pieds des plants. Elle se faisait à la volée par des 
personnes âgées, surtout pour la technique en planches. La première application de NPK était effectuée entre le $6^{\text {ème }}$ et le $22^{\text {ème }}$ jour après le repiquage des plants d'oignon. La fumure organique était appliquée entre le $10^{\text {ème }}$ et le $14^{\text {ème }}$ jour après la première application du NPK, s'il y a lieu, à la dose théorique de $20 \mathrm{t} / \mathrm{ha}$.

Une seconde application d'engrais chimique, composée d'un mélange de NPK et d'urée, était réalisée dans la période comprise entre le $17^{\text {ème }}$ le $25^{\text {ème }}$ jour après l'application de la fumure organique. Les doses variaient entre 500 et $700 \mathrm{~kg} / \mathrm{ha}$ pour le NPK et 400 à $500 \mathrm{~kg} / \mathrm{ha}$ pour l'urée. Le coût moyen de la main d'œuvre pour l'épandage de l'engrais était de $14285 \mathrm{FCFA} / \mathrm{ha} \pm 1425$ pour 3 producteurs suivis.

\section{Sarclage}

Le sarclage était pratiqué manuellement par les femmes âgées, car c'est une opération qui doit être réalisée avec soin pour ne pas arracher les racines des plantules. Il consiste à arracher les hautes herbes et ensuite, à l'aide de binettes et de dabas, les femmes remuent le sol afin d'améliorer la structure superficielle du sol. La fréquence de cette opération dépend de la vitesse de repousse et de la densité des mauvaises herbes. Pour 5 producteurs suivis, le coût moyen de la main d'œuvre était de 40 164 FCFA /ha \pm 6371 .

\section{Récolte}

La récolte intervient à la maturité des bulbes, entre le $90^{\text {ème }}$ et le $120^{\text {ème }}$ jour après repiquage, selon la variété. Cette maturité est caractérisée par un ramollissement du collet et le flétrissement des feuilles. L'opération était réalisée en plusieurs étapes par les femmes, comme suit:

- déterrage

Il consiste à arracher manuellement et à déterrer les bulbes restés en terre à l'aide de petites pioches. Le temps moyen de l'opération était de $25 \mathrm{~h} / \mathrm{ha} \pm 5$.

- effeuillage

L'effeuillage est réalisé avec des couteaux artisanaux sur un support en bois qui sert de support pour trancher le collet. Le temps moyen de travail était de $42 \mathrm{~h} / \mathrm{ha} \pm 6$.

- conditionnement

Après avoir rassemblé les bulbes sur une aire aménagée en bordure du champ, le conditionnement était fait dans des sacs en jute, surmontés d'un filet, avant la couture des bords. Deux hommes tiennent le sac et le secouent lors du remplissage par les femmes. Le temps moyen du conditionnement était de $15 \mathrm{~h} / \mathrm{ha} \pm 2$. La sommation des temps de travaux par opération unitaire de la récolte donnait un temps de récolte de $82 \mathrm{~h} / \mathrm{ha} \pm 13$. La main d'œuvre féminine de la récolte est rémunérée en nature par les feuilles obtenues de l'effeuillage. Elles pilent ces feuilles fraîches, forment des boules qu'elles sèchent. Ces boules sont utilisées pour la cuisine en période creuse de l'oignon bulbe. Les hommes qui participent au conditionnent sont pris en charge par l'acheteur du produit.

\section{Coûts moyens des productions de l'oignon bulbes}

Les charges de production par opération ont été déterminées par l'enquête et par le suivi. Il n'existait pas de différences de coûts des opérations obtenues par l'enquête et par le suivi. Toutefois, il a été considéré le prix des intrants importés sur le marché. Le Tableau 1 résume les charges de production obtenues à l'hectare. Les frais de mise en place et d'entretien de la pépinière ne sont pas pris en compte.

Le coût de la main d'œuvre de la récolte étant rémunéré par les feuilles collectées par les femmes, n'est pas pris en compte dans les charges de production à l'hectare. 
Tableau 1: Charges de production d'oignon autour du barrage de Goinré dans le Yatenga au Burkina Faso.

\begin{tabular}{lcccc}
\hline Activités & Quantité & Unité & $\begin{array}{c}\text { Coût } \\
\text { unitaire(FCFA) }\end{array}$ & $\begin{array}{c}\text { Coût total } \\
\text { (FCFA/ha) }\end{array}$ \\
\hline Semence & 1 & ha & 237500 & 237500 \\
Défrichage/traitement herbicide & 1 & ha & 23560 & 23560 \\
Labour en traction bovine & 1 & ha & 28571 & 28571 \\
Emiettement/nivellement & 1 & ha & 35714 & 35714 \\
Billonnage & 1 & ha & 39796 & 39796 \\
Pesticide & 1 & ha & 18000 & 18000 \\
Repiquage & 1 & ha & 51038 & 51038 \\
Sacs de 50 kg de NPK & 14 & Sacs/ha & 18000 & 252000 \\
Fumure organique & 57 & Charretées/ha & 1000 & 57000 \\
Transport fumure & 57 & voyage/ha & 500 & 28500 \\
Epandage fumure & 1 & ha & 14286 & 14286 \\
Sarclage & 1 & ha & 40164 & 40164 \\
Sacs de 50 kg d'urée & 9,5 & Sacs/ha & 17000 & 161500 \\
Irrigation & 1 & ha & 21450 & 21450 \\
Total & & & $\mathbf{1 0 0 9 ~ 0 7 9}$ \\
\hline
\end{tabular}

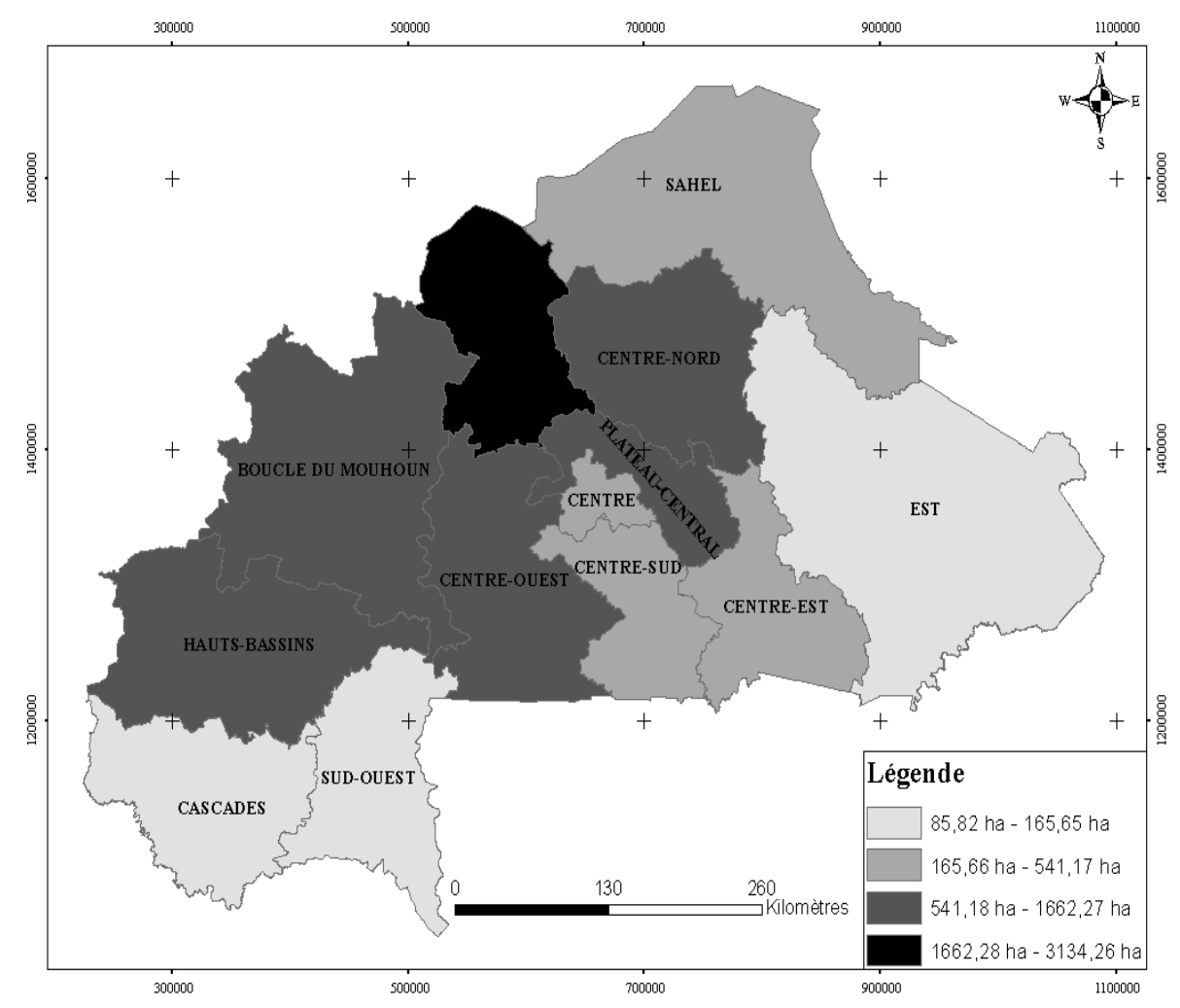

Figure 1: Zones de production de l'oignon en fonction des superficies emblavées au Burkina Faso. Source : Direction de la prospective et des statistiques agricoles et alimentaires, 2011. 


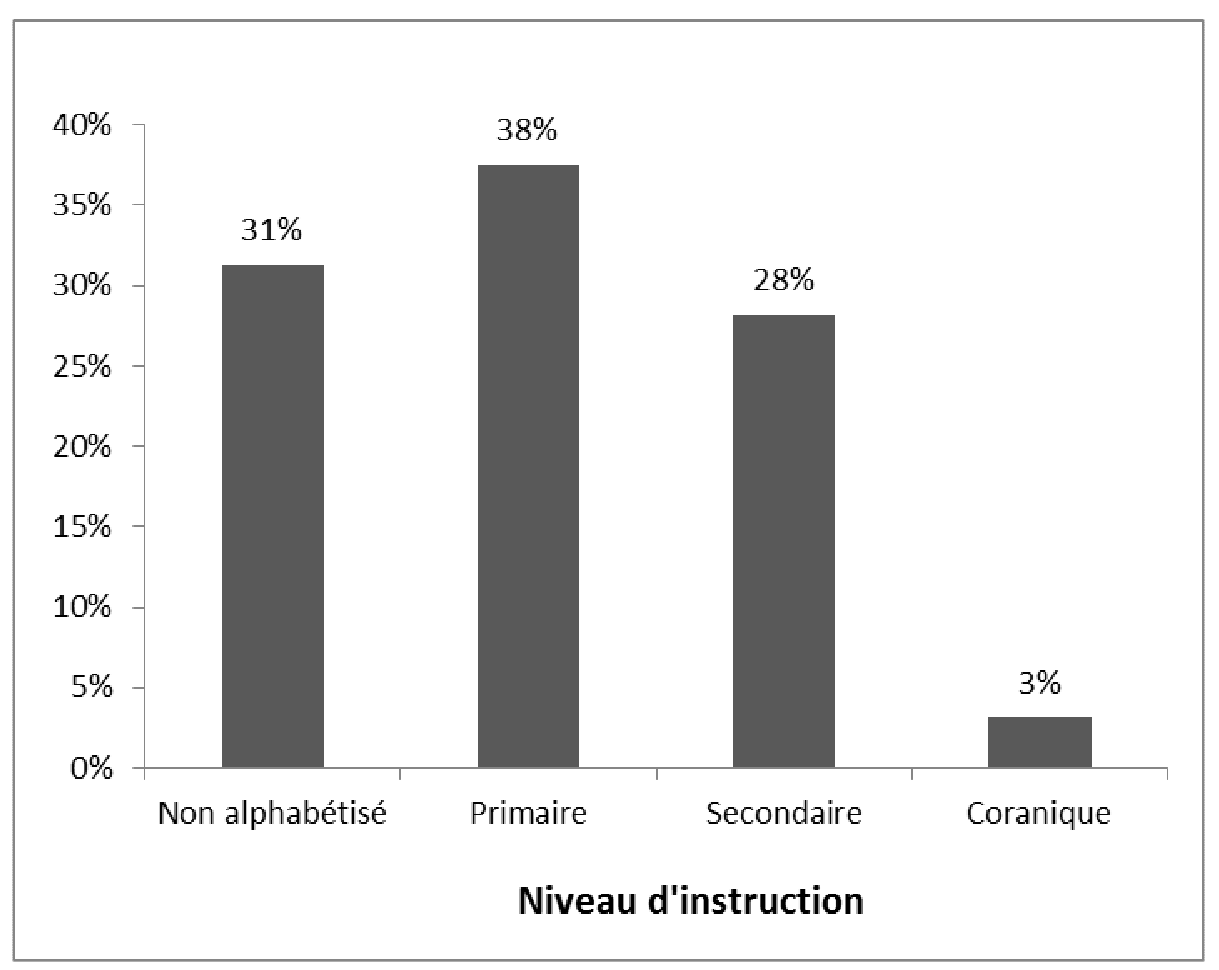

Figure 2: Niveau d'éducation des producteurs d'oignon sur les sites d'étude.

\section{DISCUSSION}

La production de l'oignon bulbe est une activité de contre saison pour les populations des régions productrices de ce légume au Burkina Faso. Cette production contribue à réduire l'exode rural des jeunes et procure des revenus aux femmes qui ont leur propre parcelle de production, même si leurs superficies sont plus réduites que celles des hommes. Le taux d'analphabétisme élevé (31\%) des producteurs, est compensé par le nombre d'années d'expérience de ces derniers dans la production de l'oignon. Ce constat confirme celui de Ouédraogo (2008), qui a montré que les producteurs du Yatenga avaient une bonne expérience dans cette activité. Cependant, la stabilité des superficies exploitées est menacée par des difficultés d'accès aux terres qui se caractérisent par des locations, des empreints de terre, avec des incertitudes d'une année à l'autre. D'ailleurs, cette observation est confortée par le fait que le niveau de mécanisation n'a pas d'influence directe sur la superficie exploitée, mais plutôt le statut de propriétaire terrien par la lignée familiale. Les emprunts sont justifiés par le fait que les propriétaires veulent bénéficier de l'arrière effet des fertilisants utilisés pour la production de l'oignon. Aussi, les producteurs maraîchers assistent gratuitement les propriétaires de terre dans la récolte de leurs céréales, afin de libérer rapidement la parcelle à emprunter.

A l'exception du labour, toutes les opérations de la production sont manuelles, du fait que l'offre en outils appropriés pour les productions maraîchères n'est pas disponible sur le marché ou est méconnue par les acteurs. Ce niveau primaire de la mécanisation a un impact sur la productivité de l'oignon et sa compétitivité sur le marché sous-régional comme le soulignent Alessandro et Alseny (2008). Cette contrainte sera d'autant plus élevée que la main d'œuvre devient de plus en plus rare et de plus en plus chère avec la scolarisation devenue de plus en plus obligatoire et avec l'explosion des sites miniers qui attirent la main d'œuvre, surtout la 
frange jeune de la population. Des opérations, comme le repiquage mobilise une main d'œuvre importante (301 h/ha \pm 6$)$, il devient nécessaire de moderniser les outils de production, au risque de voir les productions baissées au fil des années. Aussi, au regard des faibles superficies individuelles exploitées, il importe de développer la prestation de services mécanisés pour faciliter l'accès à la mécanisation à tous les acteurs.

Des opérations de production de l'oignon, seul le labour est mécanisé. En traction bovine, ce temps moyen était de 14 $\mathrm{h} / \mathrm{ha} \pm 4$; ce qui n'est pas significativement différent des résultats de Son (2004) qui a trouvé une moyenne de $17 \mathrm{~h} / \mathrm{ha}$ en production cotonnière.

Toutefois, les variations des temps de travaux sont élevées pour les opérations réalisées manuellement. En effet, il est observé que les temps de travaux sont d'autant plus élevés que le nombre de personnes sur la parcelle dépasse 3 .

$\mathrm{Au}$ regard des temps de travaux obtenus, la priorité de la mécanisation des opérations pourrait porter sur le nivellement/émiettement, le billonnage, le repiquage et le déterrage des bulbes. En termes de coûts, ces opérations sont les plus coûteuses à l'exception du sarclage qui avait un coût moyen de 40164 FCFA/ha. Quant à la densité de plants à hectare, les écartements entre plants $(10,99 \mathrm{~cm} \pm 1,37)$ répondent à la norme recommandée par la Fédération des Producteurs Agricoles du Burkina (FEPA-B). Par contre, la moyenne des écartements entre les lignes $(16,9 \mathrm{~cm} \pm 1,27)$ diffère de celle recommandée par la FEPA-B, qui varie de 30 à $40 \mathrm{~cm}$ (FEPA-B, 2007). Cette différence d'écartements entre lignes est liée aux équipements et techniques de billonnage utilisés.

Le système d'irrigation à la raie, avec le transport de l'eau à l'aide de tubes PVC mobiles, engendre des pertes énormes en eau tant au niveau du transport qu'à la parcelle. Pourtant, les producteurs ont relevé l'insuffisance d'eau pour boucler le cycle de l'oignon dans les années de mauvaise pluviométrie, ce qui les amène à réduire les superficies. Son (2009) a signalé que les expériences du Mali, dans les régions de Mopti et de San ont donné des résultats satisfaisants. Le système d'irrigation goute à goute pourrait être une alternative à l'irrigation à la raie. Aussi, l'introduction du goutte à goutte pourrait s'accompagner du pompage solaire, cela réduirait la hantise des pannes fréquentes des motopompes au cours du cycle de production et dont le coût de fonctionnement (réparation et entretien) reste relativement élevé. Toutefois, les fréquences d'irrigation doivent être alliées au besoin de la plante en fonction de son stade de développement.

L'utilisation d'environ 5 formules d'engrais, montre qu'il n'y pas de formule spécifique à l'oignon. Les producteurs disent n'utiliser la fumure organique que lorsqu'elle est disponible.

La commercialisation de l'oignon est contrôlée individuellement et non par les organisations paysannes en bord champ. Aussi, ce sont les clients qui livrent les sacs de conditionnement dont le prix par sac est négocié avec le producteur. Il importe d'uniformiser le matériel de conditionnement et de faire la promotion de la vente en poids en lieu et place des ventes en volume dont les quantités ne sont pas maîtrisées.

Il résulte de ce qui suit que la compétitivité de l'oignon burkinabè réside dans la modernisation des outils de production et l'organisation de la commercialisation.

\section{Conclusion}

La mécanisation des opérations culturales ne se résume qu'au labour, quel que soit le niveau (traction animale ou motorisée). Les superficies exploitées dépendent plus des conditions d'accès à la terre que du niveau de mécanisation. En traction motorisée, le coût du labour était de $42857 \mathrm{FCFA} /$ ha contre 28 $571 \mathrm{FCFA} /$ ha en traction bovine.

Les opérations contraignantes se sont révélées être les opérations qui demandent beaucoup de temps en main d'œuvre et en coûts. Ce sont le repiquage (301 h/ha), le 
billonnage

l'émiettement/nivellement (66 h/ha).

Le repiquage est l'opération la plus coûteuse (51 038 FCFA/ha) en main d'œuvre. Il est suivi du sarclage (40 164 FCFA/ha), du billonnage (39 796 FCFA/ha) et de l'émiettement/nivellement (35 714 FCFA/ha). Ces coûts qui iront croissants à cause du manque de main d'œuvre contribuent à réduire la compétitivité de l'oignon bulbe du Burkina Faso.

Afin de rendre compétitive l'oignon burkinabè sur le marché régional, il est important de prioriser la mécanisation des opérations culturales, comme l'émiettement/nivellement, le billonnage, le repiquage et le déterrage, tout en organisant la commercialisation et l'accès aux terres.

\section{CONFLIT D'INTERETS}

Les auteurs déclarent qu'il n'y a pas de conflit d'intérêts entre eux.

\section{CONTRIBUTIONS DES AUTEURS}

GS est le principal investigateur; il a rédigé le protocole, supervisé la collecte et l'analyse des données et a écrit le manuscrit. RK et SGY ont mené l'enquête, contribué à la collecte et l'analyse des données ainsi qu'à la rédaction du manuscrit.

\section{REFERENCES}

Alessandro S, Alseny S. 2008. Note technique : Évaluation sous-régionale de la chaine de valeurs oignon / échalote en Afrique de l'ouest, ATP, 58p.

Direction générale des prévisions et des statistiques agricoles. 2008. Rapport, Analyse de la filière maraichage. Ouagadougou, Burkina Faso, 117p.

Direction de la prospective et des statistiques agricoles et alimentaires. 2011. Rapport d'analyse du module maraîchage. Ouagadougou, Burkina Faso, 214p.

FEPA-B. 2007. Oignon, fiches techniques, Ouagadougou, Burkina Faso, 2p.
Institut nationale des statistiques et la démographie. 2003. Rapport final de l'analyse des résultats d'enquête burkinabè sur les conditions de vie des ménages. Ouagadougou, Burkina Faso, 270p.

Ouédraogo A. 2008. Facteurs de vulnérabilité et stratégies d'adaptation aux risques des maraîchers urbains et périurbains dans les villes de Ouahigouya et de Koudougou. Mémoire d'ingénieur. Institut du Développement Rural, Université Polytechnique de Bobo-Dioulasso, Burkina Faso, 58p.

Réseau national des chambres d'agriculture, 2011. Exportations d'oignons hollandais vers l'Afrique de l'Ouest : Présentation des données commerciales en provenance d'Eurostat. Note d'information / Filière oignon $\mathrm{n}^{\circ} 10$.

Son G, Yé SG, Some MTA, Kiogo R, Dayamba KF, Ouedraogo L, Napo HI. 2014. Enquête Diagnostique des Systèmes Techniques de Production de l'Oignon. Institut de Recherche en Sciences Appliquées : Ouagadougou, Burkina Faso ; 47.

Son G. 2009. Etude sur les équipements agricoles les plus utilisés en lien avec la mécanisation agricole dans les régions de Ségou et Mopti. Programme d'appui au sous-secteur de l'irrigation de proximité, Mali, p.40.

Son G. 2004. Amélioration des techniques de préparation du sol dans les exploitations à traction animales de la zone cotonnière Ouest du Burkina Faso : développement et mise en œuvre d'un décompacteur à dents. Thèse d'Etat, Institut Agronomique et Vétérinaire HASSAN II. Rabat. Royaume du Maroc, p.118. 